\title{
A new large-diameter overtube for endoscopic submucosal dissection in the colon
}

The single-balloon overtube was initially developed for deeper insertion of the scope into the small bowel [1]. Ohya et al. [2] reported that use of the balloon overtube improved access to the lesion and facilitated scope manipulation in colorectal endoscopic submucosal dissection (ESD). Here we report our experience with a newly developed balloon overtube for colorectal ESD, especially in the right colon.

The novel balloon overtube designed for colonoscopy (ST-Y0001-3C1, Olympus, Tokyo, Japan) has an outer diameter of $15.6 \mathrm{~mm}$, inner diameter of $13.2 \mathrm{~mm}$, and total length $770 \mathrm{~mm}$. The inflated balloon is $52 \mathrm{~mm}$ long and has a diameter of $42.4 \mathrm{~mm}$. A colonoscope with water-jet function is preloaded into the overtube prior to ESD ( $\bullet$ Fig. 1). After reaching the target lesion ( $\bullet$ Fig. 2 ), the overtube is inserted under fluoroscopic guidance, enabling repeated insertion and removal of the endoscope. After a circumferential mucosal incision of the lesion has been made, the endoscope is retrieved and a short, disposable, transparent hood is attached to the endoscopic tip to make the submucosal layer more visible. The hood-mounted colonoscope is reinserted through the overtube up to the lesion. Then the overtube balloon is inflated, followed by simultaneous stretching of the scope and the overtube, which prevents inadvertent slippage of the scope ( $\bullet$ Fig. 3 ).

The average diameter of the specimens retrieved in our case series was $38.7 \mathrm{~mm}$, with an average tumor diameter of $32.4 \mathrm{~mm}$. The mean procedure time were 110 minutes. Both the complete resection rate and the en bloc resection rate was $100 \%$. One perforation occurred during the ESD, but this was managed successfully with intravenous antibiotics ( Table 1). Although further studies are required to validate our findings, the newly developed overtube allowed us to use instruments such as a magnifying colonoscope with water-jet function and hood devices, all of which facilitated the colorectal ESD.

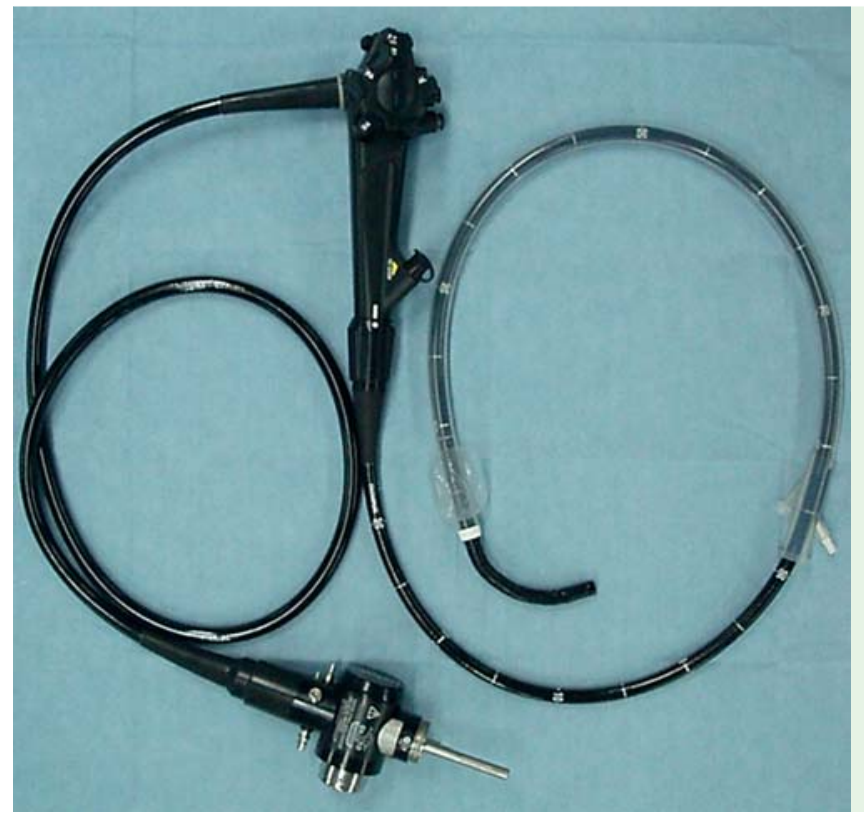

Fig. 1 Balloon overtube preloaded to the therapeutic colonoscope.

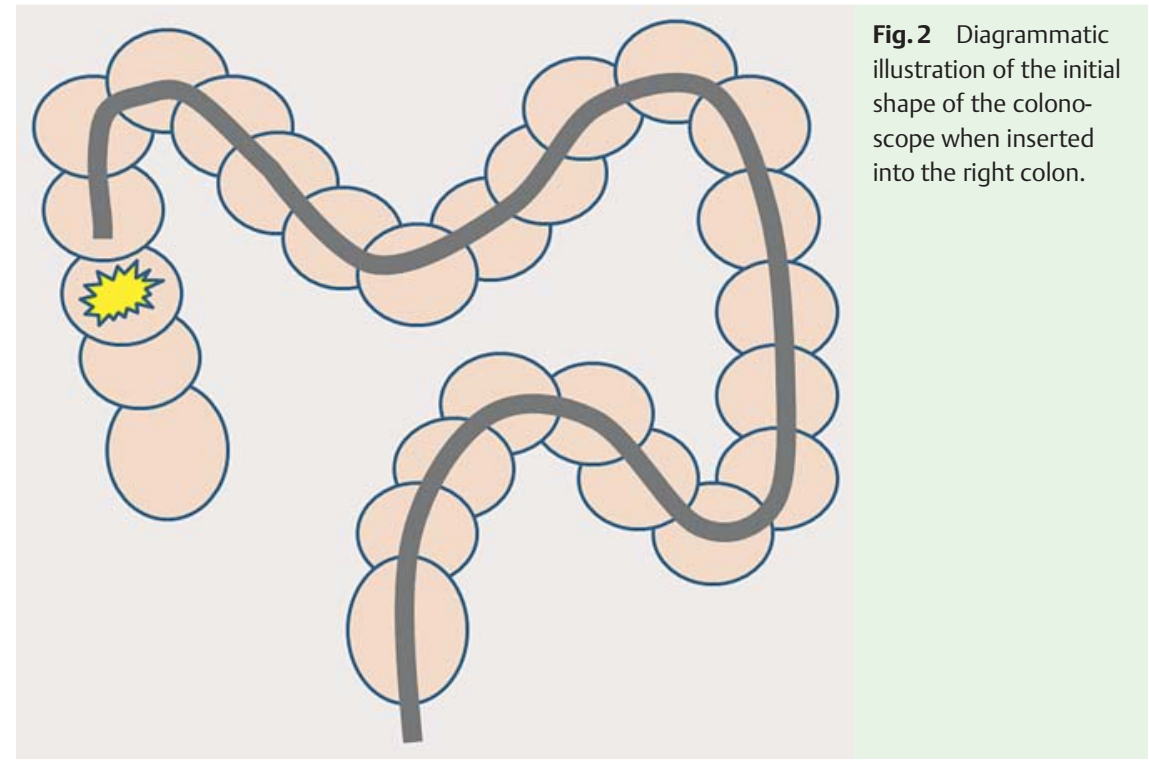

Endoscopy_UCTN_Code_TTT_1AQ_2AD

K. Okamoto, N. Muguruma,

S. Kitamura, Y. Fujino, T. Goji,

H. Yano, T. Kimura, H. Miyamoto, T. Okahisa, T. Takayama

Department of Gastroenterology and Oncology, Institute of Health Biosciences, University of Tokushima Graduate School, Tokushima City, Japan 


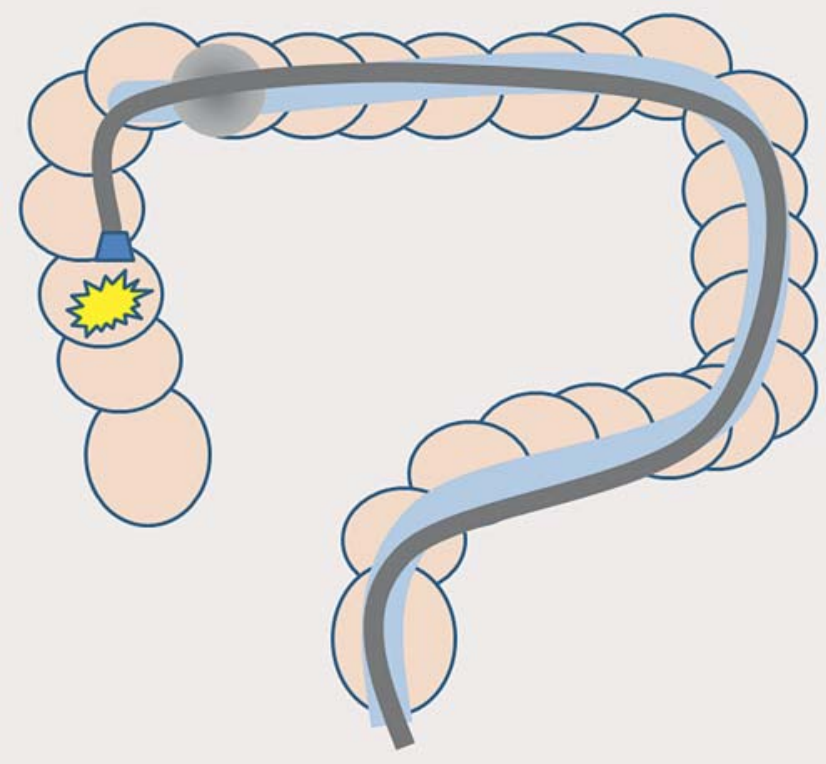

Fig.3 Diagrammatic illustration of the straightened colon with the single balloon overtube and reinserted colonoscope attached with a hood.

\section{References}

1 Kawamura T, Yasuda K, Tanaka K et al. Clinical evaluation of a newly developed singleballoon enteroscope. Gastrointest Endosc 2008; 68: $1112-1116$

2 Ohya T, Ohata K, Sumiyama K et al. Balloon overtube-guided colorectal endoscopic submucosal dissection. World J Gastroenterol 2009; 15: 6086-6090

\section{Bibliography}

DoI http://dx.doi.org/

10.1055/s-0032-1310249

Endoscopy 2012; 44: E395-E396

(c) Georg Thieme Verlag KG

Stuttgart · New York

ISSN 0013-726X

\section{Corresponding author \\ N. Muguruma}

Department of Gastroenterology and Oncology Institute of Health Biosciences

University of Tokushima Graduate School

3-18-15, Kuramoto-cho

Tokushima City 770-8503

Japan

Fax: +81-88-6339235

muguruma.clin.med@gmail.com

Table 1 Patient characteristics and results of endoscopic submucosal dissection using an overtube. All the lesions were completely resected.

\begin{tabular}{|c|c|c|c|c|c|c|c|c|c|}
\hline Patient & Sex & $\begin{array}{l}\text { Age } \\
\text { (years) }\end{array}$ & $\begin{array}{l}\text { Location of the } \\
\text { lesion }\end{array}$ & $\begin{array}{l}\text { Specimen size } \\
(\mathrm{mm})\end{array}$ & $\begin{array}{l}\text { Lesion size } \\
(\mathrm{mm})\end{array}$ & $\begin{array}{l}\text { Length of pro- } \\
\text { cedure (min) }\end{array}$ & Bleeding & Perforation & $\begin{array}{l}\text { Histological } \\
\text { diagnosis }\end{array}$ \\
\hline 1 & $\mathrm{~F}$ & 72 & Ascending colon & 27 & 25 & 60 & - & - & $\begin{array}{l}\text { Carcinoma } \\
\text { in adenoma }\end{array}$ \\
\hline 2 & M & 62 & Cecum & 30 & 28 & 120 & - & + & $\begin{array}{l}\text { Serrated } \\
\text { adenoma }\end{array}$ \\
\hline 3 & $\mathrm{~F}$ & 64 & Ascending colon & 50 & 42 & 120 & - & - & Adenoma \\
\hline 4 & M & 65 & Ascending colon & 40 & 37 & 100 & - & - & $\begin{array}{l}\text { Carcinoma } \\
\text { in adenoma }\end{array}$ \\
\hline 5 & $\mathrm{~F}$ & 77 & Transverse colon & 30 & 21 & 70 & - & - & Carcinoma \\
\hline 6 & M & 63 & Ascending colon & 55 & 50 & 100 & - & - & $\begin{array}{l}\text { Serrated } \\
\text { adenoma }\end{array}$ \\
\hline 7 & M & 75 & Cecum & 58 & 24 & 120 & - & - & Carcinoma \\
\hline 8 & M & 76 & Transverse colon & 40 & 35 & 100 & - & - & $\begin{array}{l}\text { Carcinoma } \\
\text { in adenoma }\end{array}$ \\
\hline 9 & M & 79 & Splenic flexure & 50 & 45 & 120 & - & - & $\begin{array}{l}\text { Carcinoma } \\
\text { in adenoma }\end{array}$ \\
\hline 10 & M & 62 & Transverse colon & 40 & 35 & 100 & - & - & Adenoma \\
\hline 11 & $\mathrm{~F}$ & 85 & Cecum & 30 & 25 & 110 & - & - & Adenoma \\
\hline 12 & M & 80 & Splenic flexure & 30 & 25 & 120 & - & - & Adenoma \\
\hline 13 & M & 56 & Ascending colon & 22 & 20 & 110 & - & - & Adenoma \\
\hline 14 & $\mathrm{~F}$ & 72 & Ascending colon & 50 & 45 & 210 & - & - & Adenoma \\
\hline 15 & M & 71 & Cecum & 15 & 13 & 90 & - & - & $\begin{array}{l}\text { Carcinoma } \\
\text { in adenoma }\end{array}$ \\
\hline 16 & M & 76 & Ascending colon & 50 & 45 & 120 & - & - & $\begin{array}{l}\text { Carcinoma } \\
\text { in adenoma }\end{array}$ \\
\hline 17 & M & 59 & Splenic flexure & 40 & 35 & 100 & - & - & $\begin{array}{l}\text { Carcinoma } \\
\text { in adenoma }\end{array}$ \\
\hline
\end{tabular}

